\title{
Large-area crack-free single-crystal photonic crystals via combined effects of polymerization-assisted assembly and flexible substrate
}

\author{
Jinming Zhou ${ }^{1}$, Jingxia Wang ${ }^{1}$, Yu Huang ${ }^{1,2}$, Guoming Liu ${ }^{1}$, Libin Wang ${ }^{1,2}$, Shuoran Chen ${ }^{1,2}$, Xiuhong Li $^{3}$, \\ Dujin Wang ${ }^{1}$, Yanlin Song ${ }^{1}$ and Lei Jiang ${ }^{1}$
}

Cracking of photonic crystals (PCs) has received considerable attention because of its severe limitation to PC's applications in high-performance optics devices. Although enormous efforts have been focused on the understanding and elimination of the uncontrolled cracks in the self-assembly process, no reliable, low cost and scalable methods have been demonstrated for the fabrication of large (cm or more) crack-free single-crystalline PCs. Herein, we present a facile, reliable approach for the assembly of crack-free single-crystalline PCs on the centimeter scale by the synergistic effects of substrate deformation and monomer infiltration/polymerization. The co-assembling monomer infiltrates and polymerizes in the interstices of the colloidal spheres to form an elastic polymer network, which could lower the tensile stress generated from colloid shrinkage and strengthen the long-range interactions of the colloidal spheres. Otherwise, the timely transformation of the flexible substrate releases the residual stress. This facile, scalable and environment-friendly approach to centimeter-scale crack-free singlecrystalline PCs will not only prompt the practical applications of PCs in high-performance optics devices, but also have great implications for the fabrication of crack-free thin films in other fields, such as wet clays, coating and the ceramic industry. NPG Asia Materials (2012) 4, e21; doi:10.1038/am.2012.38; published online 3 August 2012

Keywords: crack-free; large area; photonic crystals; self-assembly; single crystal

\section{INTRODUCTION}

Photonic crystals (PCs) have aroused worldwide attention because of their special light manipulation properties, which are extensively exploited in integrated optical devices, ${ }^{1-3}$ full-color displays ${ }^{4,5}$ and chemical/biological sensors. ${ }^{6-8}$ Self-assembly of submicrometer colloidal spheres into PC structures is a promising approach due to its simplicity and low cost, compared with top-down lithography techniques. ${ }^{9-11}$ However, the unintended crack formation in the selfassembly process has severely hindered the fabrication of large-area single crystalline PCs and their corresponding applications in highquality optics devices. ${ }^{12-14}$ Accordingly, much attention has been focused on the cracking mechanism ${ }^{15,16}$ and elimination. ${ }^{17-25}$ Generally, cracking is mainly caused by the tensile stress generated in the self-assembly process, which arises from the shrinkage of the colloidal spheres during the final drying process and the constraint of this shrinkage by the rigid substrate. ${ }^{15,16}$ Based on this understanding, various approaches have been devised to eliminate the tensile stress and avoid cracking. ${ }^{17-25}$ Firstly, preventing the colloidal spheres from shrinking by preshrinking them before assembly is an effective approach to eliminate the tensile stress and avoid cracking. ${ }^{19}$ Secondly, using the liquid substrate (molten Ga) ${ }^{21}$ or patterned substrate $^{24}$ allows the homogeneous shrinkage of the colloidal spheres, thus avoiding the stress production during the assembly process. Last, but not least, the preferential selection of the lowstacking density crystal structure is favorable for the elimination of cracking as it releases the stress from the interstices between the lattice faces. As a typical example, Aizenberg et al. ${ }^{25}$ prepared large-area crack-free PCs with a low-stacking density (110) lattice structure. However, the aforementioned approaches are either tedious ${ }^{22}$ or involve using poisonous ${ }^{21}$ and costly substrates, ${ }^{24}$ and the largest single crystalline domain ever-reported is not more than $200 \mu \mathrm{m} \times 200 \mu \mathrm{m} .{ }^{18}$ No reliable, low cost, scalable methods have been demonstrated for the fabrication of large ( $\mathrm{cm}$ or more) crack-free single-crystal PCs.

Herein, we present a facile strategy for large-area crack-free singlecrystalline PCs by the combined effects of polymerization-assisted assembly and substrate deformation. The co-assembling monomers infiltrate and polymerize in the interstices of the colloidal spheres,

${ }^{1}$ Beijing National Laboratory for Molecular Sciences (BNLMS), Laboratory of New Materials, Key Laboratory of Organic Solids, Laboratory of Advanced Polymer Materials, Institute of Chemistry, Chinese Academy of Sciences, Beijing, PR China; ${ }^{2}$ Graduate University of Chinese Academy of Sciences, Beijing, PR China and ${ }^{3}$ Shanghai Synchrotron Radiation Facility, Shanghai Institute of Applied Physics, Chinese Academy of Sciences, Shanghai, China

Correspondence: Dr J Wang or Professor Y Song, Laboratory of New Materials, Institute of Chemistry, Chinese Academy of Sciences, Zhongguancun North First Street 2, Beijing 100190, PR China.

E-mail: wangzhang@iccas.ac.cn or ylsong@iccas.ac.cn

Received 6 March 2012; revised 24 May 2012; accepted 12 June 2012 
which could reduce the shrinkage of the colloidal spheres and lower the generated tensile stress. The infiltrated polymer also strengthens the long range interactions of colloidal spheres based on its elasticity. Otherwise, the timely transformation of the flexible substrate releases the residual stress. Elastic polymer infiltration and substrate deformation result in an integration of micrometer-sized single-crystal domains into centimeter scale crack-free single-crystalline PCs, which are the largest ever-reported. The obtained PCs show an improved optical property due to the large-area well-ordered single-crystalline structure. This facile approach to large-area single-crystalline PCs will be promising for PCs' practical applications in high-quality optical integrated devices, optical waveguides and so on.

\section{MATERIALS AND METHODS}

\section{Materials}

Metal foils with different thickness were obtained from Kaihua Aluminum Company limited, Jiangsu, China. These foils were cleaned in 1:1 absolute ethanol and acetone ultrasonically before use. Monodisperse latex spheres of poly(styrene-methyl methacrylate-acrylic acid) (poly(St-MMA-AA)) were synthesized via our previous method ${ }^{26-28}$ and were directly used without further treatment. $N$-isopropyl acrylamide (NIPAm) was purchased from Tokyo Chemical Industry Co, Ltd. $N, N^{\prime}$-methylenebisacrylamide (99\%) and ammonium persulfate (98\%) were purchased from Sigma-Aldrich (Shanghai, China). All the reagents were purified by re-crystallization before use.

\section{Methods}

Four percent monomer solution was prepared by mixing $0.64 \mathrm{~g}$ monomer, $2.9 \mathrm{~g} 1 \% \mathrm{~N}, \mathrm{~N}^{\prime}$-methylenebisacrylamide, $0.26 \mathrm{~g} 1 \%$ ammonium persulfate into $12.2 \mathrm{~g}$ of water. The monomer solution was then mixed with poly(St-MMAAA) colloidal dispersion with the weight ratios of monomer/colloid as 0:1, $0.5: 1,1: 1,1.5: 1$ and 2:1. After dispersing ultrasonically for $30 \mathrm{~min}$, the suspension was divided into vials of $5 \mathrm{ml}$. Aluminium (Al) foils were vertically positioned in these vials. Subsequently, the monodispersed poly(St-MMA-AA) colloidal spheres were self-assembled on the $\mathrm{Al}$ foils at $60^{\circ} \mathrm{C}$ and relative humidity of $60 \%$. During the assembly process, the monomer infiltrated in the interstices of the poly(St-MMA-AA) colloidal spheres and polymerized with $N$, $N^{\prime}$-methylenebisacrylamide crosslinker under the initiation of ammonium persulfate, and upon drying, crack-free composite opal PCs were obtained. Moreover, the inverse opal PCs without cracks were facilely obtained after dissolving the poly(St-MMA-AA) colloidal spheres with 1,4-dioxane. In these experiments, monomers used are NIPAm, acrylic acid, acrylamide, crotonyl alcohol, trans-2-butenioc acid, 2-hydroxyethyl methacrylate and 2-(dimethylamino)ehyl methacrylate.

\section{Characterization}

Scanning electronic microscopy (SEM) images were obtained with a fieldemission SEM microscope, JSM-6700, JEOL, Ltd (Tokyo, Japan). Ultravioletvis spectra were recorded with an Ocean Optic HR 4000 fiber optic ultravioletvis spectrometer (Dunedin, FL, USA), normal to the (hkl, miller indices of a notation system in crystallography for planes and directions in crystal lattice $)=(111)$ planes of the crystal. Synchrotron small-angle X-ray scattering (SAXS) measurement was performed at the beamline BL16B1 at Shanghai Synchrotron Radiation Facility, China, using grazing-incidence SAXS (GISAXS) due to the presence of the substrate. The energy of the X-ray radiation was $10 \mathrm{keV}$, resulting in a wavelength of $0.124 \mathrm{~nm}$. The size of the primary X-ray beam was $1 \times 1.2 \mathrm{~mm}^{2}$ with the incident angle of the X-ray to the sample of $c a 2^{\circ}$ and the sample-to-detector distance was $5240 \mathrm{~mm}$. The size of the X-ray beam at the sample position was $28 \times 1 \mathrm{~mm}^{2}$ for GISAXS. For SAXS data collection, a Mar165 CCD detector (Diamond Light Source, Oxfordshire, UK) with an average pixel size of $80 \times 80 \mu \mathrm{m}^{2}$ was used. The SAXS data were corrected for background scattering and normalized with respect to the primary beam intensity. The yield strength $\left(R_{p 0.2}\right)$ of metal foils with different thicknesses was tested at $20^{\circ} \mathrm{C}$ using a domestic electronic universal tensile tester (WDW-10E, Time Group, Nanjing, China) controlled by a computer at a tensile test rate of $1.5 \mathrm{~mm} \mathrm{~min}^{-1}$. The yield point was determined by the $0.2 \%$ offset technique. Thermal characteristics were measured using a differential scanning calorimeter (differential scanning calorimetry, TA Instruments, Q2000, 45, New Castle, DE, USA).

\section{RESULTS AND DISCUSSIONS}

\section{Characterization of large-area crack-free single-crystalline PCs}

The fabrication of large-area crack-free single-crystalline PCs is shown in Scheme 1. Monodisperse colloidal spheres of poly(St-MMA-AA) are co-assembled with a water-soluble monomer (NIPAm for instance) on metal foils. While the colloidal spheres self-assemble into well-ordered periodic structures, the monomer infiltrates and polymerizes in the interstices of the colloidal spheres to form a crosslinked elastic polymer network. The crosslinked polymer network can fix the perfectly-ordered latex arrangement in the saturated dispersion, favoring the achievement of large-area crack-free single crystalline PCs. Meanwhile, the used flexible substrate of metal foils can be deformed to release the residual stress. The synergetic effects of elastic polymer infiltration and the substrate deformation allow the achievement of large-area crack-free composite opal and inverse opal PCs.

The colloidal spheres of poly(St-MMA-AA) synthesized in our lab were chosen because they are favorable for the fabrication of large area crack-free single crystalline PCs. Firstly, the colloidal sphere of

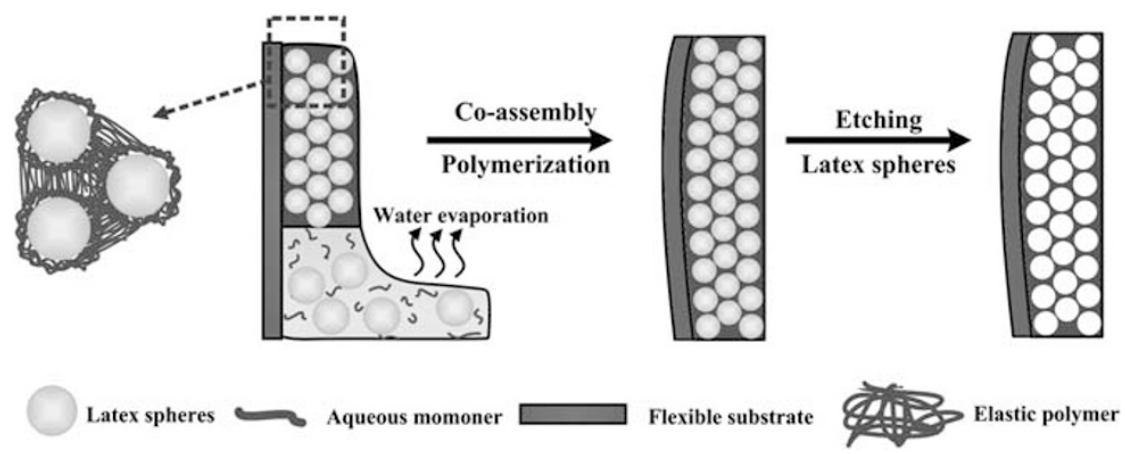

Scheme 1 Fabrication process for crack-free photonic crystals (PCs) by polymerization-assisted assembly on aluminium foil. In the assembly process, the monomer polymerizes and forms an elastic polymer in the interstices of the colloidal spheres. The elastic deformation of the as-formed polymer counteracts the volume change resulted from latex shrinkage and decreases the tensile stress generated. Meanwhile, the substrate deformation releases the residual stress. Both contribute to the achievement of crack-free single-crystalline PCs. 

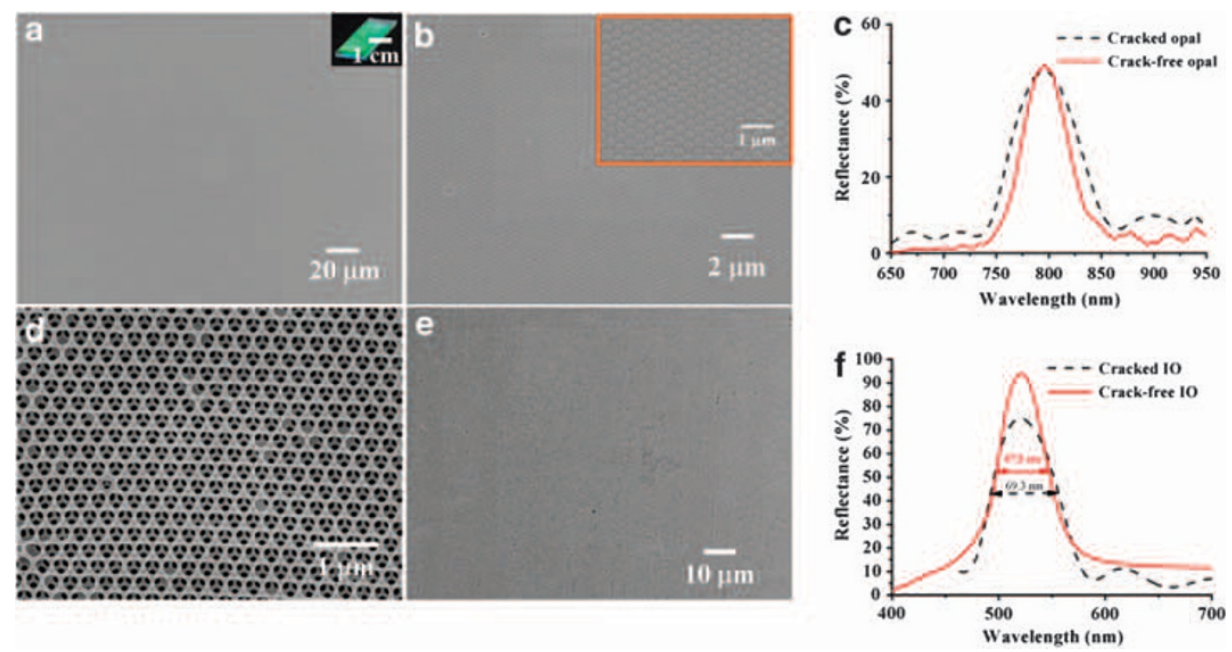

Figure 1 Scanning electronic microscopy images, ultraviolet-vis spectra of the crack-free PNIPAm/colloid composite opal (a-c) and poly $N$-isopropyl acrylamide inverse opal (d-f) photonic crystals (PCs) assembled on Al foils. The inset in (a) is a digital photograph of crack-free PC taken at an angle of ca $45^{\circ}$ from the sample surface, and the scale bar is $1 \mathrm{~cm}$. The inset in $(\mathbf{b})$ is the magnified SEM image. These SEM images indicate that cracks have been completely eliminated from the PCs. Narrower full-width-at-half-maximum is observed for the crack-free colloidal PCs compared with that of cracked PCs.

poly(St-MMA-AA) has good monodispersity with a polydispersity index of 0.005 . The excellent monodispersity is beneficial for the large-scale assembly of uniform PCs. Secondly, the colloidal spheres have a special core-shell structure with a hydrophilic PAA shell and a hydrophobic PS core. The hydrogen bonding among carboxyl groups of the hydrophilic shell is beneficial for the well-ordered colloidal assembly. Thirdly, the hydrophilic shell is helpful for the infiltration of the hydrophilic co-assembling monomer, such as NIPAm or acrylamide, into the interstices of the colloidal particles, favoring their homogeneous infiltration.

Figures $1 \mathrm{a}$ and $\mathrm{b}$ present the SEM images of as-prepared polyNIPAm (PNIPAm)-poly(St-MMA-AA) composite opal PCs assembled on $20-\mu \mathrm{m}$-thick $\mathrm{Al}$ foil. Clearly, PCs with an area of $1.5 \times 2 \mathrm{~cm}^{2}$ (Figures $1 \mathrm{a}$ and $\mathrm{b}$, Supplementary Figure $\mathrm{S} 1$ and Supplementary Figure S2) are devoid of cracks. As-prepared composite opal PCs are highly periodic over the whole sample with (111) close-packed faces parallel to the substrates (Figure 1b). Obviously, the interstices of poly(St-MMA-AA) colloidal spheres have been fully and uniformly infiltrated, as is observed in the magnified SEM image inserted in Figure 1b. PNIPAm infiltration reduces the shrinkage of the colloidal spheres and strengthens the interaction of the colloidal spheres, which favors the crack elimination ${ }^{20}$ and the integration of micrometer sized single-crystalline domains into centimeter-scale single-crystalline PCs. The large area crack-free PCs were assembled with latex diameters of 320,180, 230 and $260 \mathrm{~nm}$ (Figure $1 \mathrm{~b}$ and Supplementary Figure S2). Furthermore, after selectively dissolving the colloidal spheres with 1, 4-dioxane, crack-free PNIPAm inverse opal PCs are also obtained, as shown in Figures1d and e. The absence of cracks in the (111) plane, which is most prone to cracking, ${ }^{29}$ implies the complete elimination of cracks in the as-prepared PCs.

Expectedly, the obtained large-area crack-free PNIPAm-poly (St-MMA-AA) composite opal PCs show a single-crystalline property determined by GISAXS, which is a nondestructive tool to characterize the macroscopic single crystal structure of PCs. ${ }^{30-33}$ Figure 2 (Supplementary Figures S3a, b) presents the GISAXS result of the as-prepared large-area crack-free PCs. The incident beam was parallel to the (111) plane, and thus the collected data were from $<110\rangle$ or $<11 \overline{2}>$ zones. The data exhibit typical lattice diffraction features in accordance with the simulated pattern of the $\langle 01 \overline{1}\rangle$ zone in Supplementary Figure S3b, indicating a single-crystalline structure. ${ }^{30-33}$ As the size of the primary X-ray beam at the sample position was $c a 28 \mathrm{~mm} \times 1 \mathrm{~mm}$ for the GISAXS experiment, it could be assumed that the single crystal array in Figure $2 \mathrm{~b}$ reveals macroscopic single-crystal domains on the centimeter scale. ${ }^{33}$ The spacing of $d_{200}$ and $d_{100}$ is obtained from the diffraction data: $\mathrm{d}_{200}=2 \pi / \mathrm{q}_{(200)}=261.7 \mathrm{nmd}_{100}=2 \mathrm{~d}_{200}=523.4 \mathrm{~nm}$. Thus, a centerto-center distance $(R)$ between two adjacent particles is calculated through: $R=\mathrm{d}_{100} / \sqrt{2}=370.2 \mathrm{~nm}$. The calculated value is $50.2 \mathrm{~nm}$ higher than the latex diameter of $c a 320 \mathrm{~nm}$ observed by SEM in Figure $1 \mathrm{~b}$. The increase by $50.2 \mathrm{~nm}$ could result from the infiltration of elastic polymer into the interstice of latex particles, which is close to the average wall thickness ( $c a 45 \mathrm{~nm}$ ) of the inverse opal. The characterization of large scale single-crystalline domain is in consistence with the long-rang well-ordered crystal structure of the crackfree PCs in Figure 1b. The homogeneous PNIPAm infiltration in the interstices of the latex spheres (inserted image in Figure 1b) produces an interconnected network among latex particles and strengthens the interactions of the latex spheres.

The centimeter scale crack-free single-crystalline domain resulted in the optical improvement in Figures $1 \mathrm{c}$ and $\mathrm{f}$ (Supplementary Figure S2d). The full-width-at-half-maximum of the reflectance spectra (Figures $1 \mathrm{c}$ and $\mathrm{f}$ and Supplementary Figure S2d) of the crack-free PCs (ca $48 \mathrm{~nm}$ ) is significantly narrower (30\%) than that of the corresponding cracked PCs $(c a 70 \mathrm{~nm})$. The much narrower stopband may be due to the elimination of scattering effects caused by the cracks. ${ }^{22}$ This largest crack-free single-crystalline PCs ever-reported with improved optical properties will have potential applications in full optical circuit, next-generation displays and so forth.

\section{Effect of monomer infiltration/polymerization on the fabrication of large-area crack-free single crystalline PCs}

Proper selection of co-assembling monomer is crucial for the fabrication of large-area crack-free single-crystal PCs. Firstly, the monomer is aqueous and could easily be dispersed in the latex suspension without affecting the system's stability. Secondly, the asformed polymer is elastic, which could undergo reversible expansion/ 

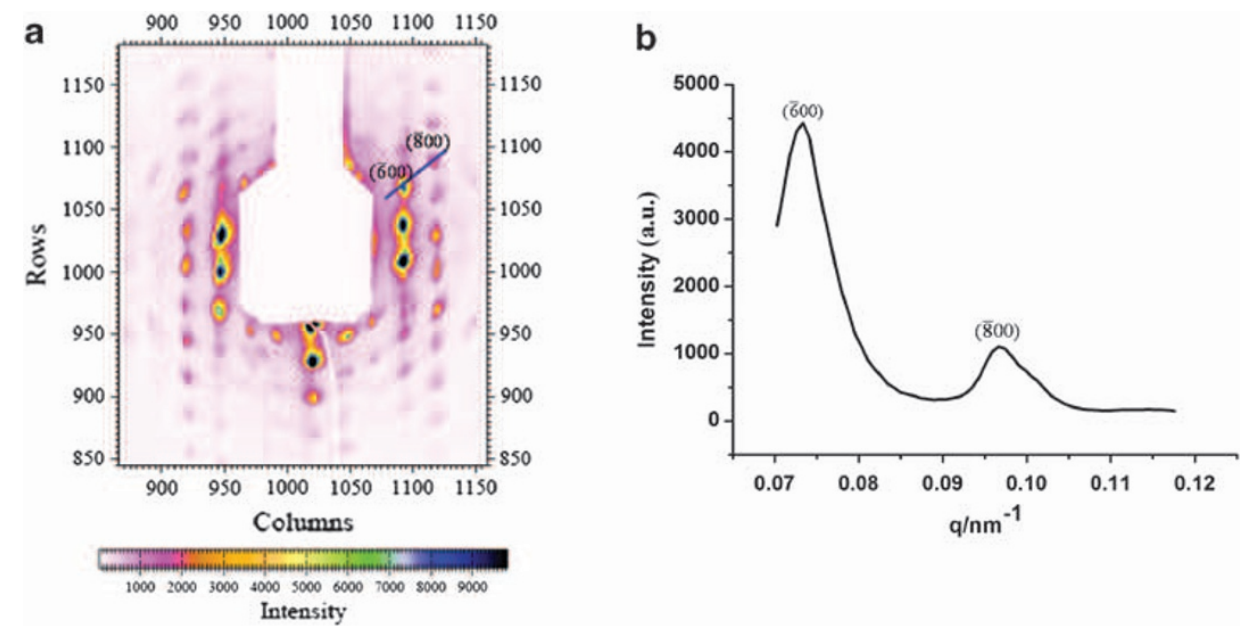

Figure 2 (a) Grazing-incidence small-angle X-ray scattering diffraction pattern of as-prepared crack-free photonic crystal (PC) assembled on Aluminium foil. (b) Line profiles taken along the blue line through the $(\overline{6} 00)$ and $(\overline{8} 00)$ in the images. The distance between $(\overline{6} 00)$ and $(\overline{8} 00)$ is equal to $q_{200}$. The diffraction pattern accords with the simulation zone of $\langle 011(-)\rangle$ zone, and the size of the primary X-ray beam at the sample position is $28 \times 1.2 \mathrm{~mm}^{2}$, which indicates the as-obtained colloidal crystal is macroscopic single-crystalline PC with centimeter scale.
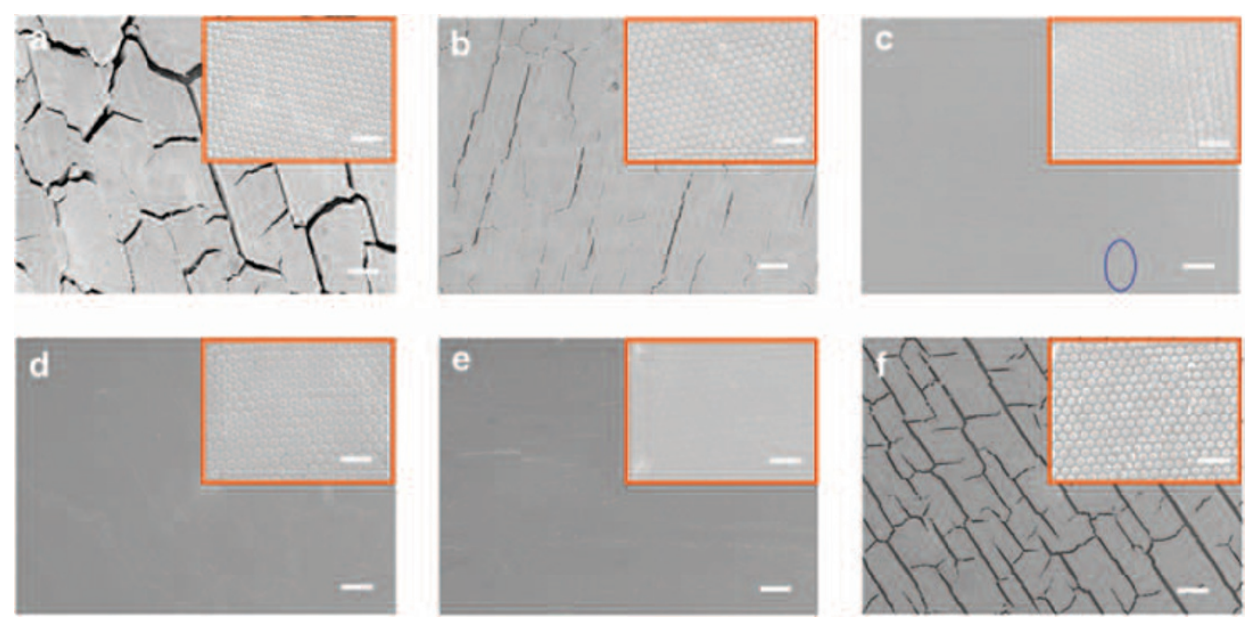

Figure 3 Scanning electronic microscopy images of poly $\mathrm{N}$-isopropyl acrylamide (PNIPAm)/colloid composite PCs assembled with varying weight ratios of NIPAm/colloid (a) 0:1, (b) 0.5:1 (c) $1: 1$, (d) $1.5: 1$, (e) $2: 1$ on Al foils of $20 \mu \mathrm{m}$ and (f) $1.5: 1$ on glass. The scale bar is $10 \mu \mathrm{m}$ (the inset is $1 \mu \mathrm{m}$ ). It indicates that the width and number of cracks decrease with the increasing infiltration of PNIPAm into the interstice of the latex particles and the optimized NIPAm/colloid ratio for crack-free PCs is $\sim 1.5: 1$.

contraction to absorb the stress during the assembling process. Thirdly, the polymerization rate of the monomer must be slower than the assembly rate of latex particles, which ensures the wellordered latex assembly and the homogeneous infiltration of the polymer into the interstice of the latex particles. Too rapid monomer polymerization will cause the viscosity growth of the system and affect the well-ordered latex assembly. Based on these considerations, aqueous monomers, such as acrylic acid, acrylamide, crotonyl alcohol, trans-2-butenioc acid, 2-hydroxyethyl methacrylate and 2-(dimethylamino)ehyl methacrylate, were used to fabricate the corresponding composite opal PCs (Supplementary Figure S4). It was found that NIPAm, acrylic acid and acrylamide were the optimal choice as infiltrating monomers. In particularly, the as-prepared crack-free PNIPAm composite PCs demonstrated the best well-ordered latex arrangement. In this case, the NIPAm polymerizes to form PNIPAm, which precipitates from the latex system because the assembly temperature is over $33^{\circ} \mathrm{C}$ (the lower critical solution temperature of PNIPAm) ${ }^{34}$ The precipitation of PNIPAm effectively avoids the viscosity growth of the system, favoring the well-ordered latex assembly. Importantly, the infiltration of PNIPAm among the latex interstice fixes the perfectly-ordered latex arrangement in the saturated dispersion ${ }^{15}$ and strengthens the latex interactions, which contributes to the large-area single crystalline PCs (Figure 2). In contrast, composite PCs from other monomers show relatively poor periodic structure although the cracks are completely eliminated. The relatively poor periodic structure could be attributed to the dramatic viscosity growth resulting from the rapid monomer polymerization, which would disturb the assembly process. 

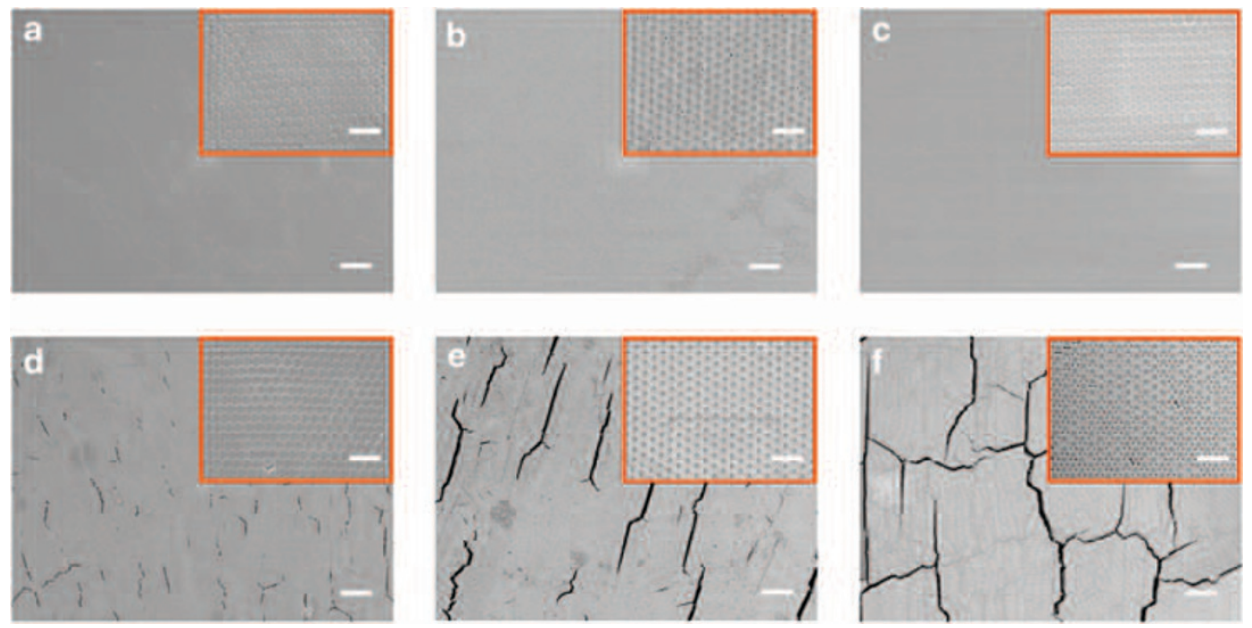

Figure 4 SEM images of PNIPAm-polystyrene-methyl methacrylate-acrylic acid composite opal photonic crystal (PCs) assembled on Al foils with varying thickness of (a) 20, (b) 65, (c) 100 , (d) 130 , (e) 180 and (f) $280 \mu \mathrm{m}$, respectively. The scale bar is $10 \mu \mathrm{m}$ (the inset red scale bar is $1 \mu \mathrm{m}$ ). Cracking is eliminated when the PNIAm-colloid composite opal PCs are assembled on aluminium (Al) foils with thickness $\leqslant 100 \mu \mathrm{m}$. In contrast, cracks are found when the films are assembled on thicker Al foils.

To determine an optimal monomer infiltration amount, a series of PCs were prepared on $20-\mu \mathrm{m}$-thick $\mathrm{Al}$ foils with varying weight ratios of monomer/colloid in the co-assembling system from $0: 1$ to $2: 1$. Taking NIPAm as an example, the crack width of as-prepared PCs decreases from $c a 3$ to 0.5 to $0.4 \mu \mathrm{m}$ (Figures $3 \mathrm{a}-\mathrm{c}$ ), as the ratios of NIPAm/colloid increase from $0: 1$ to $0.5: 1$ to $1: 1$, respectively. The optimal NIPAm/colloid ratio for crack-free PCs is 1.5:1 (Figure 3d), and an overlayer of PNIPAm occurs upon the PCs' surface (Figure 3e) when the ratio of NIPAm/colloid approaches 2:1. Meantime, an obvious red shift of the stopband of the PCs is observed with the PNIPAm/colloid ratios increasing from $0: 1$ to $0.5: 1$ to $1: 1$ (Supplementary Figure S5). The phenomenon implies that PNIPAm is evenly infiltrated into the interstices of the colloidal spheres, which results in a continuous growth of the refractive index and of the center-to-center distance $(R)$ between two adjacent particles (insert image in Figure 1b). In comparison, the shift of the stopband position becomes inconspicuous when the ratio of NIPAm/colloid is over 1:1 (Supplementary Figure S5b), which means that the interstice of the colloidal spheres is almost fully infiltrated. Accordingly, it is concluded that the critical monomer/colloid ratio for large-area crackfree PCs should be about 1:1, over which crack-free PCs could be achieved. The value is a little higher than the theoretical value of 0.85:1 (Supplementary Figure S6), which may result from the volume shrinkage as the monomer polymerizes during the assembly process.

\section{Effect of the used substrate on large-area crack-free single crystalline PCs}

A proper substrate is necessary for the fabrication of large-area singlecrystal PCs. Large cracks are still found in PNIPAm/colloid composite opal PCs assembled on a glass substrate (Figure 3f) even at the optimized infiltration ratio of $1.5: 1$. To further assess the specific requirements of the substrate for fabrication of crack-free PCs, a series of PNIPAm/colloid composite opal PCs were assembled on Al foils with different thickness at fixed NIPAm/colloid ratio of 1.5:1 and a fixed latex concentration of $0.2 \mathrm{wt} \%$ (Figure 4). Clearly, crack-free composite opal PCs were obtained on Al foils with thickness $\leqslant 100 \mu \mathrm{m}$ (that is, 20, 65 and $100 \mu \mathrm{m}$ ) (Figures $4 \mathrm{a}-\mathrm{c}$ ), while cracks occurred in the PC films on $\mathrm{Al}$ foils with thicknesses $>100 \mu \mathrm{m}$, such as 130,180 and $280 \mu \mathrm{m}$ (Figures $4 \mathrm{~d}-\mathrm{f}$ ). This could be attributed to the distinct deformation behavior of $\mathrm{Al}$ foils determined by their thickness. ${ }^{35}$ The thin Al foil deforms easily, which favors the timely release of the tensile stress generated during the assembly process. ${ }^{35}$ The intrinsic deformation behavior is based on the $R_{p 0.2}$ of different $\mathrm{Al}$ foils (in Supplementary Figure S7a). The thinner the Al foils, the lower their $R_{p 0.2}$ and the easier for the deformation of $\mathrm{Al}$ foils; consequently, the stress is easily released, which contributes to the large-area crack-free PCs (Figures $4 \mathrm{a}-\mathrm{c}$ ). Vice versa, the thicker Al foils show higher $R_{p 0.2}$, which results in a greater difficulty for their deformation and the release of the stress, leading to the cracked PCs (Figures $4 \mathrm{~d}-\mathrm{f}$ ). In our experiment, other metal substrates, such as $\mathrm{Cu}$, $\mathrm{Ti}$ and Ni foils (Supplementary Figure S8), were also applied for the fabrication of crack-free PCs. Besides Al foils, the critical thickness for fabrication of crack-free PCs with fixed latex concentration of $0.2 \%$ was also determined: Ti foil with thickness $\leqslant 35 \mu \mathrm{m}, \mathrm{Cu}$ foil with thickness $\leqslant 105 \mu \mathrm{m}$ and $\mathrm{Ni}$ foil with thickness $\leqslant 55 \mu \mathrm{m}$ (Supplementary Figure S8). Subsequently, at a fixed metal foil thickness $(100 \mu \mathrm{m}$-thick-Al-foil for instance), PCs with different thickness were assembled by varying the latex concentration and the critical crack-free PC thicknesses were determined. In the case of Al foil with a thickness of $100 \mu \mathrm{m}$, the critical crack-free PC thickness is $\sim 5.6 \mu \mathrm{m}$ assembled with a latex concentration of $0.2 \%$ (Supplementary Figure S9 and Supplementary Figure S7b). The relationship of PC thickness (cracking or not) and substrate compliance are summarized in Figure 5. The $R_{p 0.2}$ of the substrate and the critical crack-free PC thickness demonstrates typical reciprocal relation, which is in good agreement with the theoretical relationship between the critical stresses for nucleation of a crack in a film of identical elastic spheres and the critical film thickness predicted based on Griffith's energy balance concept. ${ }^{15}$ From Figure 5, the thickest crack-free PC that can be fabricated herein is $\sim 5.6 \mu \mathrm{m}$, over which cracks develops in the PCs. The value differs with the calculated critical crack-free film thickness of $\sim 19.7 \mu \mathrm{m}$ with randomly-packed colloidal spheres. ${ }^{15}$ The deviation may arise from the higher tensile stress generated in a highly ordered system and the weakness of the closest packed crystal structure. ${ }^{29}$ The specific requirement for large area crack-free PCs determined herein provides great guidance for the fabrication of crack-free PCs and has great implications for PC practical applications. 


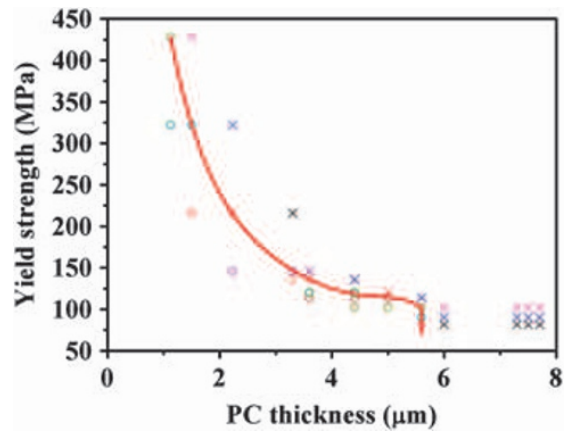

Figure 5 The cracking behaviors of photonic crystals (PCs) with varying thickness assembled on substrates with different $R_{p 0.2}(\mathrm{O}$ : crack-free PC $\times$ : cracked $\mathrm{PC}$ ). The $R_{p 0.2}$ of the substrate and the critical crack-free PC thickness demonstrates typical reciprocal relation and the thickest critical crack-free PC that can be fabricated is $\sim 5.6 \mu \mathrm{m}$, over which cracks develops in the PCs.

It should be mentioned that water has an important role in the fabrication process of the large area crack-free single-crystal PCs, due to the hydrophilic property of the latex spheres. Water adsorbed in the PNIPAm hydrogel makes the composite PCs more robust, which decreases the tensile stress during the assembly process through reducing latex shrinkage. The slower water evaporation form the PNIPAm hydrogel ensures the timely release of the generated tensile stress through substrate deformation, avoiding its accumulation to cause cracks. The elastic property of the infiltrated polymer among the latex spheres strengthens their interaction as to form large area single-crystalline PCs without cracks. However, after complete drying in the programmable constant temperature/humidity machine, the materials scratched off from the $\mathrm{Al}$ foils were characterized by differential scanning calorimetry and thermogravimetric analysis. No characteristic peaks of ice melting at $\sim 0{ }^{\circ} \mathrm{C}$, related to water sorption, ${ }^{36}$ or thermal degradation at $\sim 110^{\circ} \mathrm{C}$, related to water loss in the thermogravimetric analysis curve, ${ }^{37}$ were observed (Supplementary Figure S10), indicating the water content in the obtained composite PC was negligible. This negligible water content favors the practical applications of PCs at elevated temperatures. After treating the PCs at temperature from $120^{\circ} \mathrm{C}$ to $180^{\circ} \mathrm{C}$, no obvious degradation of optical property was found when the film was treated at 120 , 140 and $160^{\circ} \mathrm{C}$ for $1 \mathrm{~h}$. In contrast, crack formation and reflectance intensity drop were observed after the film was treated at $180^{\circ} \mathrm{C}$. The crack-free PCs can be applied at an operating temperature as high as $160^{\circ} \mathrm{C}$ (Supplementary Figure S11a, b), favoring their practical applications.

\section{Mechanism for crack elimination}

The crack-avoiding mechanism is clarified in Figure 6, taking the critical crack-free PC thickness assembled on $\mathrm{Al}$ foils with a critical thickness of $100 \mu \mathrm{m}$ whose $R_{p 0.2}$ is $\sim 102.2 \mathrm{MPa}$, for instance. The crack-free PCs could be achieved when substrates with $R p_{0.2} \leqslant 102.2$ $\mathrm{MPa}$ were used and the weight ratio of monomer/colloid was over the critical value of 1:1 (Supplementary Figure S12). In contrast, cracked PCs were found due to an insufficient infiltration of elastic polymer or the use of a rigid substrate (Supplementary Figure S12). Proper infiltration of elastic polymer can counteract the volume shrinkage of the colloidal spheres and strengthens their interactions. The elastic polymer could store part of the tensile stress by its elasticity, whose stretching is observed through the cracks in a cracked PC with a

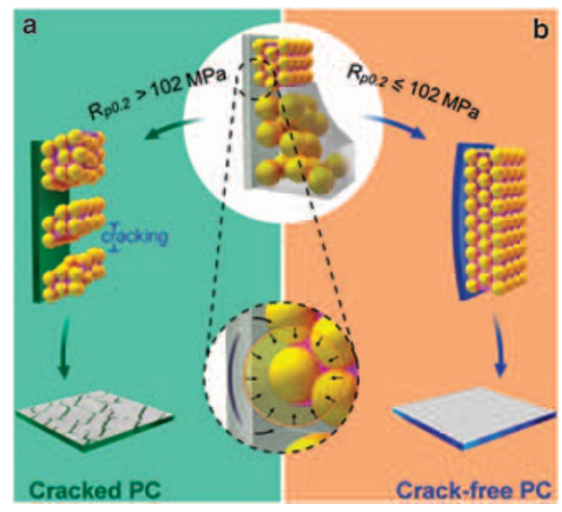

Figure 6 Schematic illustrations of the crack-avoiding mechanism. Proper poly $\mathrm{N}$-isopropyl acrylamide infiltration can minimize the tensile stress to $102 \mathrm{MPa}$ during assembly. (a) Substrates with $R p_{0.2}>102 \mathrm{MPa}$ could not release the tensile stress timely, leading to the accumulation of stress and crack formation in the photonic crystals (PCs). (b) The tensile stress generated from shrinking of colloidal spheres is released by the combined effects of polymer infiltration and substrate deformation (with $R p_{0.2} \leqslant 102 \mathrm{MPa}$ ), which contributes to the crack-free PCs.

thickness of $7.7 \mu \mathrm{m}$ (Supplementary Figure S13). The stored energy in the elastic polymer was transferred to the flexible substrate and finally released by substrate deformation, which is proved by the calculated critical tensile stress to nucleate a crack, agreeing well with the $R_{p 0.2}$ of the flexible substrate. This critical value of $R_{p 0.2}(102.2 \mathrm{MPa})$ is close to the theoretical critical tensile stress $\left(\sigma_{c}\right)(110.6 \mathrm{MPa})$ for the nucleation of a crack, as is calculated by the Griffith's energy balance concept (Supplementary Figures S9). ${ }^{15}$ The deformation of substrates with $R_{p 0.2} \leqslant 102 \mathrm{MPa}$ contributes to the timely release of the residual tensile stress, avoiding stress accumulation and subsequent cracking in PCs (Figure 6b). In contrast, the residual stress cannot be effectively released by the deformation of the substrate with $R_{p 0.2}>102 \mathrm{MPa}$, leading to a cracked PC. (Figure 6a).

In conclusion, we demonstrate a facile, reliable and general approach for the fabrication of crack-free single-crystalline PCs with centimeter scale by the synergistic effects of substrate deformation and monomer infiltration/polymerization. The critical thickness of crackfree PCs is $\sim 5.6 \mu \mathrm{m}$, below which crack-free PCs can be fabricated on a proper substrate. This facile approach to large-area single-crystalline PCs will be of great significance for PCs' applications in high-quality optics devices. Furthermore, this simple strategy for crack-free PCs may provide important insight for crack-free coatings in other areas, such as ceramics and wet clays.

\section{CONFLICT OF INTEREST}

The authors declare no conflict of interest.

\section{ACKNOWLEDGEMENTS}

This work is supported by the National Nature Science Foundation (Grant No's. 50973117, 50903089, 51173190, 91127029, 21074139, 20904061 and 20721061), and the 973 Program (2007CB936403 and 2009CB930404, 2011 CB932303 and 2011CB808400). We thank the beneficial discussion from Professor Jian Yu and Professor Zhaoxia Guo at Tsinghua University and the kind help on Small-angle X-rays test from Dr Yuzhu Wang and Dr Ping Zhou at Shanghai Institute of Applied Physics. 
1 Ishizaki, K. \& Noda, S. Manipulation of photons at the surface of three-dimensional photonic crystals. Nature 460, 367-370 (2009).

2 Nelson, E. C. \& Braun, P. V. Photons and electrons confined. Nat. Photonics 2, 650-651 (2008).

3 Kubo, S., Gu, Z. Z., Takahashi, K., Fujishima, A., Segawa, H. \& Sato, O. Tunable photonic band gap crystals based on a liquid crystal-infiltrated inverse opal structure. J. Am. Chem. Soc. 126, 8314-8319 (2004).

4 Braun, P. V. Colour without colouran. Nature 472, 423-424 (2011).

5 Arsenault, A. C., Puzzo, D. P., Manners, I. \& Ozin, G. A. Photonic-crystal full-colour displays. Nat. Photonic 1, 468-472 (2007).

6 Burgess, I. B., Mishchenko, L., Hatton, B. D., Kolle, M., Loncar, M. \& Aizenberg, J. Encoding complex wettability patterns in chemically functionalized 3D photonic crystals. J. Am. Chem. Soc. 133, 12430-12432 (2011).

7 Li, Y. Y., Cunin, F., Link, J. R., Gao, T., Betts, R. E., Reiver, S. H., Chin, V., Bhatia, S. N. \& Sailor, M. J. Polymer replicas of photonic porous silicon for sensing and drug delivery applications. Science 299, 2045-2047 (2003).

8 Sun, C., Zhao, X. W., Zhao, Y. J., Zhu, R. \& Gu, Z. Z. Fabrication of colloidal crystal beads by a drop-breaking technique and their application as bioassays. Small 4, 592-596 (2008).

9 Jiang, P. \& McFarland, M. J. Large-scale fabrication of wafer-size colloidal crystals, macroporous polymers and nanocomposites by spin-coating. J. Am. Chem. Soc. 126, 13778-13786 (2004).

$10 \mathrm{Kim}, \mathrm{S}$. H., Lee, S. Y., Yang, S. M. \& Yi, G. R. Self-assembled colloidal structures for photonics. NPG Asia Mater. 3, 25-33 (2011).

11 Ding, T., Song, K., Clays, K. \& Tung, C. H. Fabrication of 3D photonic crystals of ellipsoids: convective self-Assembly in magnetic field. Adv. Mater. 21, 1936-1940 (2009).

12 Vlasov, Y. A., Bo, X. Z., Sturm, J. C. \& Norris, D. J. On-chip natural assembly of silicon photonic bandgap crystals. Nature 14, 289-293 (2001).

13 Blanco, A., Chomski, E., Grabtchak, S., Ibisate, M., John, S., Leonard, S. W., Lopez, C., Meseguer, F., Míguez, H., Mondia, J. P., Ozin, G. A., Toader, O. \& van Driel, H. M. Large-scale synthesis of a silicon photonic crystal with a complete three-dimensional bandgap near 1.5 micrometres. Nature 405, 437-440 (2000).

14 Zhang, J. H., Li, Y. F., Zhang, X. M. \& Yang, B. Colloidal self-assembly meets nanofabrication: from two-dimensional colloidal crystals to nanostructure arrays. Adv. Mater. 22, 4249-4269 (2010)

15 Singh, K. B. \& Tirumkudulu, M. S. Cracking in drying colloidal films. Phys. Rev. Lett 98, 218302 (2007)

$16 \mathrm{Xu}$, P., Mujumdar, A. S. \& Yu, B. Drying-induced cracks in thin film fabricated from colloidal dispersions. Dry. Technol. 27, 636-652 (2009).

17 Wong, S., Kitaev, V. \& Ozin, G. A. Colloidal crystal films: advances in universality and perfection. J. Am. Chem. Soc. 125, 15589-15598 (2003).

18 Zheng, Z. Y., Gao, K. Y., Luo, Y. H., Li, D. M., Meng, Q. B., Wang, Y. R. \& Zhang, D. Z. Rapidly infrared-assisted cooperatively self-assembled highly ordered multiscale porous materials. J. Am. Chem. Soc. 130, 9785-9789 (2008).

19 Chabanov, A. A., Jun, Y. \& Norris, D. J. Avoiding cracks in self-assembled photonic band gap crystals. Appl. Phys. Lett. 84, 3573 (2004)

20 Wang, L. K. \& Zhao, X. S. Fabrication of crack-free colloidal crystals using a modified vertical deposition method. J. Phys. Chem. C 111, 8538-8542 (2007).
21 Griesebock, B., Egen, M. \& Zentel, R. Large photonic films by crystallization on fluid substrates. Chem. Mater. 14, 4023-4025 (2002).

22 Kanai, T. \& Sawada, T. New route to produce dry colloidal crystals without cracks. Langmuir 25, 13315-13317 (2009).

23 Kanai, T., Sawada, T., Toyotama, A. \& Kitamura, K. Air-pulse-driven fabrication of photonic crytal films of colloids with high spectral quality. Adv. Funct. Mater. 15, 25-29 (2005).

24 Jin, C. J., McLachlan, M. A., McComb, D. W., Rue, R. M. D. L. \& Johnson, N. P. Template-assisted growth of nominally cubic (100)-oriented three-dimensional crack-free photonic crystals. Nano Lett. 5, 2646-2650 (2005).

25 Hatton, B., Mishchenko, L., Davis, S., Sandhage, K. H. \& Aizenberg, J. Assembly of large-area, highly ordered, crack-free inverse opal films. Proc. Natl. Acad. Sci. USA 107, 10354-10359 (2010).

26 Wang, J. X., Wen, Y. Q., Ge, H. L., Sun, Z. W., Zheng, Y. M., Song, Y. L. \& Jiang, L. Simple fabrication of full color colloidal crystal films with tough mechanical strength. Macromol. Chem. Phys. 207, 596-604 (2006).

27 Wang, J. X., Zhang, Y. Z., Wang, S. T., Song, Y. L. \& Jiang, L. Bioinspired colloidal photonic crystals with controllable wettability. Acc. Chem. Res. 44, 405-415 (2011).

28 Zhou, J. M., Li, H. L., Ye, L., Liu, J., Wang, J. X., Zhao, T., Jiang, L. \& Song, Y. L. Facile fabrication of tough SiC inverse opal photonic crystals. J. Phys. Chem. C 114, 22303-22308 (2010).

29 Lawn, B. \& Wilshaw, T. R. Fracture of Brittle Solid 194-211 (Cambridge University Press, Cambridge, UK (1998).

30 Thijssen, J. H. J., Petukhov, A V., 'tHart, D. C. Imhof, A ven der Werf, C. H. M. Schropp, R. E. I. \& Van Blaaderen, A. Characterization of photonic colloidal single crystals by microradian X-ray diffraction. Adv. Mater. 18, 1662-1666 (2006).

$31 \mathrm{Hu}$, S. S., Rieger, J., Lai, Y. Q., Roth, S. V., Gehrke, R. \& Men, Y. F. In-situ observation of drying process of a latex droplet by synchrotron small-angle $\mathrm{X}$-ray scattering. Macromolecules 41, 5073-5076 (2008).

32 Vos, W. L., Megens, M., van Kats, C. \& Bosecke, P. X-ray diffraction of photonic colloidal single crystals. Langmuir 13, 6004-6008 (1997).

33 Huber, P., Bunk, O., Pietsch, U., Textor, M. \& Geue, T. Grazing incidence small angle $\mathrm{x}$-ray scattering on colloidal crystals. J. Phys. Chem. B 114, 12473-12479 (2010).

34 Lin, S. Y., Chen, K. S. \& Liang, R. C. Thermal micro ATR/FT-IR spectroscopic system for quantitative study of the molecular structure of poly( $\mathrm{N}$-isopropylacrylamide) in water. Polymer 40, 2619-2624 (1999).

35 Dai, C. Y., Zhang, G. P. \& Yan, C. Size effects on tensile and fatigue behaviour of polycrystalline metal foils at the micrometer scale. Philos. Mag. 91, 932-935 (2011).

36 Ping, Z. H., Nguyen, Q. T., Chen, S. M., Zhou, J. Q. \& Ding, Y. D. States of water in different hydrophilic polymers - DSC and FTIR studies. Polymer 42, 8461-8467 (2001).

37 Zeng, K., Fang, Y. \& Zheng, S. X. Organic-inorganic hybrid hydrogels involving poly $(N$ isopropylacrylamide) and polyhedral oligomeric silsesquioxane: preparation and rapid thermoresponsive properties. J. Polym. Sci. Pol. Phys. 47, 504-516 (2009).

(c) This work is licensed under the Creative Commons Attribution-NonCommercial-No Derivative Works 3.0

Unported License. To view a copy of this license, visit http:// creativecommons.org/licenses/by-nc-nd/3.0/

Supplementary Information accompanies the paper on the NPG Asia Materials website (http://www.nature.com/am) 